\title{
Behavior of logarithmic branch cuts in the self-energy of gluons at finite temperature
}

\author{
F. T. Brandt and J. Frenkel \\ Instituto de Física, Universidade de São Paulo \\ São Paulo, SP 05315-970, BRAZIL
}

(February 28, 2018)

\begin{abstract}
We give a simple argument for the cancellation of the $\log \left(-k^{2}\right)$ terms ( $k$ is the gluon momentum) between the zero-temperature and the temperature-dependent parts of the thermal self-energy.
\end{abstract}

There have been many studies of thermal Green functions in gauge field theories [1] [7], which show that their behavior at finite temperature is rather different from the one at zero temperature. In particular, it was recently pointed out by Weldon [8] that in QED, the logarithmic branch cut singularities cancel to one loop-order, in the thermal self-energy of the electron.

The purpose of this note is to show that in the Yang-Mills theory, a somewhat similar behavior occurs in the full gluon self-energy, which includes finite temperature effects. Of course, in this theory, the massless gluons are quite modified by these effects and the gluon propagator requires the Braaten-Pisarski resummation. Nevertheless, it is interesting to remark that, even before such a procedure is carried out, the one-loop $\log \left(-k^{2}\right)$ terms cancel in the sum of the $T=0$ and the $T \neq 0$ contributions to the gluon self-energy. As we shall see, this happens because the $\log \left(-k^{2}\right)$ terms appear in the thermal part of the self-energy only in the combination $\log \left(-k^{2} / T^{2}\right)$. But one can show that the $\log \left(T^{2}\right)$ contributions have the same structure as the ultraviolet divergent terms which occur at zero temperature [9]. Consequently, the $\log \left(-k^{2} / T^{2}\right)$ terms combine directly with the $\log \left(-k^{2} / \mu^{2}\right)$ contributions which occur at $T=0$ ( $\mu$ is the renormalization scale), so that the $\log \left(-k^{2}\right)$ terms cancel in a simple way in the thermal self-energy of the gluon. The branch cut in the $\log \left(-k^{2}\right)$ contribution at $T=0$ is associated with the imaginary part of the self-energy, which gives the rate of decay of a time-like virtual gluon into two real gluons. Although this contribution cancels at $T \neq 0$, there appear then additional, temperature-dependent logarithmic branch points. These singularities indicate processes not available at zero temperature, where particles decay or are created through scattering in the thermal bath.

To one-loop order, the thermal self-energy of gluons generally depends on three structure functions, $\Pi^{T}$, $\Pi^{L}$ and $\Pi^{C}$ [10]

$$
\Pi_{\mu \nu}^{a b}\left(k_{0}, \vec{k}\right)=g^{2} C_{G} \delta^{a b}\left(\Pi^{T} P_{\mu \nu}^{T}+\Pi^{L} P_{\mu \nu}^{L}+\Pi^{C} P_{\mu \nu}^{C}\right),
$$

where the projection operators $P_{\mu \nu}^{T, L}$ are transverse with respect to the external four-momentum $k^{\mu}$ and satisfy: $k^{i} P_{i \nu}^{T}=0$ and $k^{i} P_{i \nu}^{L} \neq 0$ [6]: 7 . The non-trnsverse projection operator $P_{\mu \nu}^{C}$ can be written in the plasma rest frame as follows [10]

$$
P_{\mu \nu}^{C}=\frac{1}{k^{2}}\left[\frac{k_{\nu}}{|\vec{k}|}\left(k_{0} k_{\mu}-\eta_{\mu 0} k^{2}\right)+\mu \leftrightarrow \nu\right] .
$$

Although $\Pi^{C}$ vanishes at $T=0$ because of the Slavnov-Taylor identity, it is in general a non-vanishing function of the temperature, so that $k^{\mu} \Pi_{\mu \nu} \neq 0$ for the exact self-energy.

We will discuss here, for definiteness, the retarded thermal self-energy of the gluon, which is obtained by the analytic continuation $k_{0} \rightarrow k_{0}+i \epsilon$. (A rather similar analysis can be made in the case of the time-ordered self-energy, following the approach presented in reference [11]). In order to illustrate in a simple way the mechanism of the cancellation of the $\log \left(-k^{2}\right)$ contributions, let us first consider the special case of the Feynman gauge, where $\Pi^{C}$ vanishes even at finite temperature. Then, $\Pi^{T}$ and $\Pi^{L}$ can be expressed in the plasma rest frame in terms of linear combinations of $\Pi_{\mu}^{\mu}$ and $\Pi_{00}$. After performing the integration over the internal energies $q_{0}, \Pi_{\mu}^{\mu}$ and $\Pi_{00}$ can be written as an integral over internal on-shell momenta $q=(|\vec{q}|, \vec{q})$, as follows

$$
\Pi_{\mu}^{\mu a b}=g^{2} C_{G} \delta^{a b}\left(\frac{T^{2}}{3}-10 k^{2} I_{0}\right)
$$

and

$$
\Pi_{00}^{a b}=2 g^{2} C_{G} \delta^{a b}|\vec{k}|^{2}\left(I_{0}+4 I_{1}\right) .
$$

where ( $x$ is the cosine of the angle between $\vec{k}$ and $\vec{q}$ ). 


$$
I_{0,1}=\frac{\mu^{\epsilon}}{(2 \pi)^{3-\epsilon}} \int \frac{d^{3-\epsilon} \vec{q}}{2|\vec{q}|}\left(\frac{1}{k^{2}+2 q \cdot k}+\frac{1}{k^{2}-2 q \cdot k}\right)\left[\frac{\vec{q}^{2}}{k^{2}}\left(1-x^{2}\right)\right]^{0,1}\left[\frac{1}{2}+N\left(\frac{|\vec{q}|}{T}\right)\right] .
$$

The two terms in the last square bracket are associated respectively with the $T=0$ and the $T \neq 0$ contributions $(N$ is the Bose-Einstein distribution).

In order to express the integrations in (5) in terms of known functions, it is convenient to define the variable

$$
K(x)=\frac{1}{4 \pi i} \frac{k^{2}}{k_{0}-|\vec{k}| x} .
$$

Then it is straightforward to show that

$$
I_{0}=\frac{i \pi}{|\vec{k}|}\left(\frac{1}{4 \pi}\right)^{\frac{3-\epsilon}{2}} \frac{\mu^{\epsilon}}{\Gamma\left(\frac{3-\epsilon}{2}\right)} \int_{K_{-}}^{K_{+}} d K \int_{0}^{\infty} d|\vec{q}| \frac{|\vec{q}|^{1-\epsilon}}{|\vec{q}|^{2}+(2 \pi K)^{2}}\left[\frac{1}{2}+N\left(\frac{|\vec{q}|}{T}\right)\right],
$$

where

$$
K_{ \pm} \equiv K( \pm 1)=\frac{k_{0} \pm|\vec{k}|}{4 \pi i}
$$

The above form shows that the integrals appearing in the calculation of the gluon self-energy can be naturally expressed in terms of the quantities $K_{ \pm}$(which are proportional to the light-cone momenta $k_{0} \pm k_{3}$, if one chooses, for example, the third axis along $\vec{k}$ ).

The $|\vec{q}|$ integration of $T=0$ part of $I_{0}$, gives

$$
\frac{i}{8 \pi|\vec{k}|} \int_{K_{-}}^{K_{+}} d K\left[\frac{1}{\epsilon}-\log \frac{2 \sqrt{\pi} K}{\mu}-\frac{\gamma}{2}+1\right] .
$$

Using the fact that $\operatorname{Re} K(x)>0$, the $|\vec{q}|$ integration of the $T \neq 0$ part of $I_{0}$ (where we may set $\epsilon=0$ ), yields the result $[12$ ]

$$
\frac{i}{8 \pi|\vec{k}|} \int_{K_{-}}^{K_{+}} d K\left[\frac{T}{2 K}+\log \frac{K}{T}-T \frac{d}{d K} \log \Gamma\left(1+\frac{K}{T}\right)\right]
$$

where the logarithm of the gamma function is analytic when $K \rightarrow 0$. Then, the approximation

$$
N\left(\frac{|\vec{q}|}{T}\right)=\frac{1}{\exp (|\vec{q}| / T)-1} \simeq \theta(T-|\vec{q}|)\left(\frac{T}{|\vec{q}|}-\frac{1}{2}\right),
$$

would simply lead, after performing the $|\vec{q}|$ integration in equation $(\nabla)$, to the first two terms in the exact expression

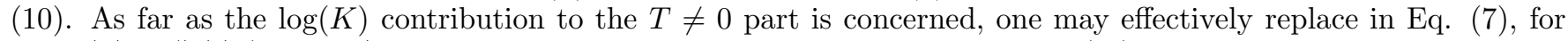
small $|\vec{q}|, N(|\vec{q}| / T)$ by $-1 / 2$. Consequently, this contribution will cancel the $\log (K)$ term associated with the $T=0$ part of $I_{0}$ (this cancellation can also be explicitly verified from Eqs. (9) and (10)).

By itself, the $K$-integration of the $\log (K / T)$ term in Eq. (10) gives the contribution

$$
\begin{gathered}
\frac{i}{16 \pi|\vec{k}|}\left[\left(K_{+}+K_{-}\right) \log \frac{K_{+}}{K_{-}}+\left(K_{+}-K_{-}\right) \log \frac{K_{+} K_{-}}{T^{2}}-2\left(K_{+}-K_{-}\right)\right]= \\
\frac{1}{32 \pi^{2}}\left[\frac{k_{0}}{|\vec{k}|} \log \frac{k_{0}+|\vec{k}|}{k_{0}-|\vec{k}|}+\log \frac{-k^{2}}{16 \pi^{2} T^{2}}-2\right] .
\end{gathered}
$$

The emergence of the $\log \left(-k^{2}\right)$ term in the special combination $\log \left(-k^{2} / T^{2}\right)$, is a direct consequence of the fact that the integrand in Eq. (10) depends only on the dimensionless ratio $K / T$. Similarly, the $\log (K / \mu)$ term in Eq. (9) yields a contribution which, apart from sign, can be obtained from Eq. (12) by the replacement $T \rightarrow \mu$. Consequently, the $\log \left(-k^{2}\right)$ terms will cancel between the zero-temperature and the temperature-dependent contributions, leaving a net factor proportional to $\log \left(\mu^{2} / T^{2}\right)$. After calculating the contributions from the first and third terms in Eq. (10), we obtain the following result for the temperature-dependent part of $I_{0}$ : 


$$
I_{0}(T)=\frac{1}{32 \pi^{2}} \log \frac{\mu^{2}}{T^{2}}+\frac{i T}{16 \pi|\vec{k}|} \log \frac{k_{0}+|\vec{k}|}{k_{0}-|\vec{k}|}+\frac{T}{8 \pi i|\vec{k}|} \log \frac{\Gamma\left(1+K_{+} / T\right)}{\Gamma\left(1+K_{-} / T\right)}
$$

Next, consider the $I_{1}$ integral which can be written as:

$$
\begin{aligned}
I_{1}=\frac{i \pi}{|\vec{k}|^{3}}\left(\frac{1}{4 \pi}\right)^{\frac{3-\epsilon}{2}} \frac{\mu^{\epsilon}}{\Gamma\left(\frac{3-\epsilon}{2}\right)} & \int_{K_{-}}^{K_{+}} d K\left[\frac{k^{2}}{(4 \pi K)^{2}}-\frac{i k_{0}}{2 \pi K}-1\right] \\
& \times \int_{0}^{\infty} d|\vec{q}||\vec{q}|^{1-\epsilon}\left[1-\frac{(2 \pi K)^{2}}{|\vec{q}|^{2}+(2 \pi K)^{2}}\right]\left[\frac{1}{2}+N\left(\frac{|\vec{q}|}{T}\right)\right] .
\end{aligned}
$$

Note that the $T=0$ contribution, associated with the factor of 1 in the second square bracket, would apparently lead to a quadratically divergent integral, which however vanishes in the dimensional regularization scheme. On the other hand, this factor yields a leading thermal contribution which is quadratic in the temperature

$$
I_{1}^{\text {lead }}(T)=\frac{T^{2}}{24|\vec{k}|^{2}}\left(1-\frac{k_{0}}{2|\vec{k}|} \log \frac{k_{0}+|\vec{k}|}{k_{0}-|\vec{k}|}\right)
$$

The $|\vec{q}|$-integration of the second term in the second square bracket of Eq. (14) is identical to the one which occurs in $I_{0}$, so that it gives analogous $\log (K)$ contributions which cancel between the $T=0$ and thermal parts. As we have seen, only such contributions would give rise, after the $K$-integration, to individual $\log \left(-k^{2}\right)$ terms. It is possible to evaluate exactly all other temperature-dependent contributions to $I_{1}$, in terms of logarithmic functions and of Riemann's zeta functions with arguments $\left(1+K_{ \pm} / T\right)$, which are analytic when $K_{ \pm} \rightarrow 0$ [11]. Since the complete expression is rather involved, we indicate here, for simplicity, only the logarithmic temperature-dependent contributions to $I_{1}$ :

$$
I_{1}^{\log }(T)=-\frac{1}{192 \pi^{2}} \log \frac{\mu^{2}}{T^{2}}-\frac{i T k^{2}}{64 \pi|\vec{k}|^{3}} \log \frac{k_{0}+|\vec{k}|}{k_{0}-|\vec{k}|} .
$$

In a general gauge, $\Pi_{\mu}^{\mu}$ and $\Pi_{00}$ will have a similar behavior (in particular, the leading $T^{2}$ contribution is gauge independent). In this case, the thermal contributions to $\Pi_{C}=k_{\mu} \Pi_{0}^{\mu} /|\vec{k}|$ are non-vanishing, and can be written as $[13]$

$$
\Pi_{C}=\frac{(1-\xi)}{(2 \pi)^{3}|\vec{k}|} \int \frac{d^{3} \vec{q}}{|\vec{q}|}\left[\left(\frac{k^{2}}{k^{2}+2 k \cdot q}+\frac{1}{2} \frac{d}{d q_{0}} \frac{k \cdot q}{q_{0}}\right) \frac{k \cdot q k_{0}-k^{2} q_{0}}{k^{2}+2 k \cdot q} N\left(\frac{\left|q_{0}\right|}{T}\right)+q \leftrightarrow-q\right]_{q_{0}=|\vec{q}|},
$$

where $\xi$ is the gauge parameter ( $\xi=1$ in the Feynman gauge) and the derivative $d / d q_{0}$ acts on all terms at its right. Performing the $|\vec{q}|$ integration, the terms involving $\log (K)$ factors turn out to be proportional to

$$
\int_{K_{-}}^{K_{+}} d K \log K\left[8 \pi i K-3 k_{0}-\frac{k^{2} k_{0}}{16 \pi^{2} K^{2}}\right]
$$

However, the coefficient of the $\log \left(-k^{2}\right)$ term, which is obtained after the $K$-integration is performed, actually vanishes:

$$
\left(2 \pi i K_{+}^{2}-\frac{3}{2} k_{0} K_{+}-\frac{k^{2} k_{0}}{32 \pi^{2} K_{+}}\right)-\left(2 \pi i K_{-}^{2}-\frac{3}{2} k_{0} K_{-}-\frac{k^{2} k_{0}}{32 \pi^{2} K_{-}}\right)=0
$$

Thus, the full self-energy of the gluon, which includes the thermal effects, does not contain $\log \left(-k^{2}\right)$ contributions. Essentially, these effects replace the zero-temperature $\log \left(-k^{2} / \mu^{2}\right)$ term by a $\log \left(T^{2} / \mu^{2}\right)$ contribution. Although this correspondence seems plausible, it is not so obvious. For instance, it would not hold if the thermal contributions would involve individual terms like $\log \left(k_{0}^{2} / T^{2}\right), \log \left(|\vec{k}|^{2} / k^{2}\right)$, etc. To discard this possibility, it is necessary to show that the $\log \left(-k^{2}\right)$ and $\log \left(T^{2}\right)$ contributions appear in the thermal part only in the combination $\log \left(-k^{2} / T^{2}\right)$. Furthermore, in order to explain the cancellation of the $\log \left(-k^{2}\right)$ terms between the zero-temperature and the temperature-dependent parts, one must also argue [9] that the $\log (T)$ dependence of the self-energy is simply related to its ultraviolet behavior at zero-temperature. Here, these properties of the thermal gluon self-energy have been explicitly verified to one-loop order. 


\section{ACKNOWLEDGMENTS}

We would like to thank CNPq (Brazil) for a grant and Prof. J. C. Taylor for a helpful correspondence.

[1] H. A. Weldon, Phys. Rev. D26, 1394 (1982).

[2] E. Braaten and R. D. Pisarski, Nucl. Phys. B337, 569 (1990); B339, 310 (1990); Phys. Rev. D45, 1827 (1992).

[3] J. Frenkel and J. C. Taylor, Nucl. Phys. B334, 199 (1990); B374, 156 (1992).

[4] R. Baier, G. Kunstatter, and D. Schiff, Phys. Rev. D45, 4381 (1992), ibid R. Kobes, G. Kunstatter, and K. Mak, Phys. Rev. D45, 4632 (1992).

[5] J.-P. Blaizot and E. Iancu, Phys. Rev. D55, 973 (1997); 56, 7877 (1997).

[6] J. I. Kapusta, Finite Temperature Field Theory (Cambridge University Press, Cambridge, England, 1989).

[7] M. L. Bellac, Thermal Field Theory (Cambridge University Press, Cambridge, England, 1996).

[8] H. A. Weldon, Phys. Rev. D59, 065002 (1999).

[9] F. T. Brandt and J. Frenkel, Phys. Rev. D55, 7808 (1997).

[10] H. A. Weldon, Annals Phys. 271, 141 (1999).

[11] A. P. de Almeida, J. Frenkel, and J. C. Taylor, Phys. Rev. D45, 2081 (1992).

[12] I. S. Gradshteyn and M. Ryzhik, Tables of Integral Series and Products (Academic, New York, 1980).

[13] F. T. Brandt and J. Frenkel, Phys. Rev. D56, 2453 (1997). 